\title{
PRESENÇA DE INSETOS DA FAMÍLIA FORMICIDAE (INSECTA: HYMENOPTERA) EM AMBIENTE HOSPITALAR, NO MUNICÍPIO DE CAMPOS GERAIS, MINAS GERAIS
}

\author{
Gilmara Aparecida SILVEIRA ${ }^{1}$ \\ Micaela de Oliveira, LOURENÇO ${ }^{2}$ \\ Melissa Vieira LEITE ${ }^{3}$ \\ Edimar Agnaldo MOREIRA ${ }^{4}$
}

1. Bióloga pela Faculdade de Ciências e Tecnologias de Campos Gerais, dilmarasilvestre@hotmail.com.

2. Bióloga pela Faculdade de Ciências e Tecnologias de Campos Gerais, tamiresmicaela@ gmail.com.

3. Docente Docente no Instituto Federal de Educação, Ciência e Tecnologia - IF Sul de Minas e no Centro Superior de Ensino e Pesquisa de Machado - CESEP, tamiresmicaela@gmail.com.

4. Pós Graduando em Ecologia e Tecnologia Ambiental, Universidade Federal de Alfenas - UNIFAL-MG, edimarbio@hotmail.com.

\section{Recebido em: 22/12/2013 - Aprovado em: 15/09/2014 - Disponibilizado em: 15/12/2014}

RESUMO: A ocorrência de infecções hospitalares tem sido motivo de preocupação e estudo no mundo todo. Como as formigas presentes em ambiente hospitalar são vetores de microrganismos que causam infecções precisam ser amostradas e controladas. O presente trabalho foi realizado com o objetivo de verificar a ocorrência de formigas em ambiente hospitalar no município de Campos Gerais, MG. Para atrair as formigas foram utilizados papéis identificados, contendo mel e sardinha expostos por um período de 3 horas. Após este período os papéis foram acondicionados em sacos plásticos contendo álcool 75\%. O material foi levado para o laboratório de zoologia da Faculdade de Ciências e Tecnologias de Campos Gerais - FACICA. As formigas foram separadas das iscas e mantidas em álcool 75\%, em frascos de polietileno e posteriormente triadas para identificação. As formigas coletadas pertencem aos gêneros: Brachymyrmex, Pheidole que compõe as subfamílias Formicinae e Myrmicinae, respectivamente. A ala com maior número desses insetos foi à cozinha, seguida da enfermaria e na pediatria nenhum espécime foi coletado. A isca que apresentou maior atratividade foi à composta por sardinha. Assim, devido à possibilidade das formigas estarem associadas a microrganismos patogênicos ao ser humano e à presença de exemplares pertencentes a gêneros já relatados como vetores, ressalta-se a necessidade de estudos complementares e de estabelecer métodos adequados para o controle das mesmas nesta instituição.

PALAVRAS-CHAVE. Formigas urbanas. Hospital. Distribuição. Atratividade

ABSTRACT: The occurrence of nosocomial infections has been of concern and study worldwide. How ants present in the hospital environment are vectors of microorganisms that cause infections need to be sampled and monitored. The present study was conducted to verify the occurrence of ants in a hospital in the city of Campos Gerais, Brazil. To attract ants were used roles identified, containing honey and sardine exposed for a period of 3 hours. After this time the roles were packed in plastic bags containing $75 \%$ alcohol. The material was brought to the laboratory of Zoology, Faculty of Science and Technology Fields General - phakic. The ant baits were separated and kept in $75 \%$ alcohol in polyethylene bottles and subsequently screened for identification. Ants collected belong to the genera: Brachymyrmex, Pheidole composing the subfamilies Myrmicinae and Formicinae respectively. The ward with the highest number of these insects was the kitchen, followed by pediatrics ward and no specimen was collected. The bait that showed higher attractiveness was composed of sardines. Thus, due to the possibility of ants are associated with pathogenic microorganisms to humans and the presence of individuals belonging to genera already reported as vectors, it emphasizes the need for further studies and to establish appropriate methods for the control of this same institution.

KEYWORDS. Ants urban. Hospital. Distribution. Attractiveness.

\section{INTRODUÇÃO}

A Ordem Hymenoptera dentro da

Classe Insecta, ocupa o terceiro lugar em abundancia de espécies, ficando após a Ordem Coleoptera e Lepidoptera. Dentre os Hymenoptera, as formigas apresentam sociabilidade contrarias as abelhas e vespas 
nas quais apenas uma fração do total das espécies apresenta uma atividade social (FERNANDEZ, 2003). As formigas são consideradas organismo eusociais por apresentar características que define o comportamento social em insetos, por exemplo: sobreposição de, ao menos, duas gerações em determinado instante do desenvolvimento colonial, com indivíduos estéreis e reprodutivos, e cuidado cooperativo com a prole (WILSON, 1971).

As formigas pertencem à família, Formicidae divididas em 16 subfamílias com 296 gêneros e 12.027 espécies descritas (AGOSTI; JOHNSON 2005), além de existirem 408 fósseis. Considera-se que existam cerca de 20.000 espécies em todo o planeta, e que no Brasil existam cerca de 2.000 espécies (HÖLLDOBLER; WILSON, 1990). Por serem insetos sociais habitam quase todos ambientes terrestres e apresentam sua maior diversidade em regiões tropicais onde há condições ambientais propícias para seu desenvolvimento (BUENO; CAMPOSFARINHA, 1999).

Estes insetos apresentam mutualismo com diversos tipos de planta, fungos e outros insetos. Os vegetais servem de fonte alimentar para as formigas através de seus nectários extraflorais, fornecendo abrigo em suas estruturas. (VITAL, 2007). Graças à dominância e diversidade, as formigas ocupam praticamente todos os processos funcionais nos ecossistemas, como por exemplo, a predação, polinização, dispersão de sementes (LEVEY; BYRNE, 1993).

As formigas, por se deslocarem rapidamente, percorrem grandes áreas isto faz com que, além de serem vetores de microrganismo em ambiente hospitalar, podem comportar-se como via de dispersão de resistência no ambiente (PEÇANHA et al. 2000 apud PEREIRA; UENO, 2008 ).

Esses insetos apresentam grande diversidade de formas e comportamentos apresentando diferenças extremas de cor, tamanho, pilosidade e agressividade dentro de um mesmo gênero. O tamanho do corpo varia de 1 milimetro a mais de $4 \mathrm{~cm}$. Colônias de formigas podem reunir desde uma dezena a alguns milhões de indivíduos (HÖLLDOBLER; WILSON, 1990). Através de odor da colônia identificam as companheiras de ninhos, no reconhecimento do grupo, durante comportamento de limpeza e lambedura entre indivíduos na colônia, bem como nas secreções que estimulam a troca de alimentos (SHOREY, 1973).

As formigas em geral exercem um papel importante no ecossistema pela ação de aeração e movimentos no solo, atuando também na decomposição de substâncias orgânicas, assim contribuindo na ciclagem de nutrientes (HOLDOBLER; WILSON, 1990). As andarilhas ou tramp species, que estão em intima associação com o homem, estão disseminadas por todo o mundo (PASSERA, 1994). 
A maior parte das espécies de formiga em ambiente urbano não apresenta um comportamento agressivo intra-específico, formando normalmente uma estrutura social do tipo uni colonial, na qual operárias e rainhas deslocam-se entre ninhos sem agressão (CHEN; NONACS, 2000).

As formigas urbanas causam incômodos, como reações alérgicas às ferroadas, contaminação de alimentos por possuírem o habito de forragear sobre o lixo e esgotos (BUENO; CAMPOS-FARINHA, 1998 apud GONÇALVES et al., 2011). Artrópodes como as formigas nem sempre foram vista pela população como insetos nocivos assim como sua relação com o possível transporte de microrganismos foram investigados na década de 70; eram vistas como incômodos, pois seu aparecimento é em grande número. Não consideravam o fato de serem importantes vetores mecânicos de microrganismos (IPINZA-REGLA, 1981 apud TEIXEIRA, 2007).

A presença de formigas no ambiente hospitalar não e necessariamente falta de limpeza. Algumas espécies apresentam atratividade por material esterilizados por preferirem locais higienizados são considerados como bioindicadores de limpeza (CINTRA, 2003). As infecções hospitalares são consideradas um dos maiores problemas em hospitais públicos e privados em todo o mundo. Fato que ultimamente vem despertando grande interesse no meio científico pela grande elevação das taxas de mortalidade de pacientes hospitalizados, o que depende diretamente das condições sanitárias e da presença de vetores dos microrganismos patogênicos. Levantamentos da composição da mirmecofauna em hospitais do Brasil demonstram que esses insetos são vetores de microrganismos patogênicos, sendo alguns resistentes a antibióticos representando risco potencial de infecção em hospitais devido à sua grande dispersão no interior destes ambientes (PESQUERO et al. 2008).

Vários fatores influenciam na presença de formigas em hospitais entre eles a infraestrutura, a proximidade a residências, fatores climáticos, alterações térmicas que estimulam a dispersão desses insetos para aparelhos eletrônicos em busca de estabilidade térmica, as embalagens de medicamentos que podem trazer nichos de formigas para $o$ ambiente interno, circulação de grande número de pessoas com roupas e os objetos que podem conter nichos de formigas além de alimentos que funcionam como atrativo (TANAKA et al. 2007).

A ocorrência de infecções hospitalares tem sido motivo de preocupação e estudo no mundo todo (BEADSON, 1972). Por esse motivo as formigas em ambiente hospitalar tem despertado interesse, desde os primeiros relatos realizados por Beatson em 1972, de que são vetores de microrganismo que causam infecções intra-hospitalares (CINTRASOCOLOWSK, 2007). 
Uma agravante para a presença desses vetores foi demonstrado nos estudos de Peçanha (2000), quando verificaram que das bactérias transportadas pelas formigas, eram Gram negativas e apresentaram níveis de resistência quantitativamente e qualitativamente mais elevados que as bactérias recuperadas do ambiente, indicando as formigas como possíveis vias de dispersão de resistência dentro do ambiente hospitalar.

Várias espécies de insetos foram estudadas por serem vetores mecânicos de microrganismos patogênicos em diferentes ambientes urbanos. Estudos em hospitais brasileiros demonstram maior número de espécies de formigas quando comparada a mirmecofauna, nos mesmos ambientes, em países como os Estados Unidos, países da Europa e Chile que apresentam clima temperado (PEÇANHA, 2000).

As instalações dos hospitais, bem como as residências próximas podem conter fatores que estimulam a migração de insetos. Os ninhos de formigas podem estar vinculados a embalagens de remédios e, além disso, alguns alimentos são atrativo extra para as formigas (ZARZUELA; RIBEIRO; CAMPOSFARINHA, 2002). Desse modo, a proposta desse trabalho foi verificar a ocorrência de formigas no hospital do Município de Campos Gerais - Minas Gerais, coletar e identificar os gêneros da família Formicidae.

\section{MATERIAIS E MÉTODOS}

O estudo foi desenvolvido no Hospital do Município de Campos Gerais. Este hospital é administrada por particular, oferecendo serviços à rede Municipal e ao SUS. No hospital foram delimitados 3 pontos de coletas, Pediatria, Enfermaria e Cozinha, sendo cada um destes subdivido em 4, totalizando 12 pontos de amostragem exceto a cozinha com 3 ponto de coletas. Por a pediatria ser um local que possivelmente pode apresentar migalhas de alimentos ingeridos por crianças, torna-se um local atrativo para esses insetos. Neste ponto de coleta foi determinados 4 locais (pediatrias) de inserção dos folhas de coleta. A enfermaria foi escolhida por instalar pessoas debilitadas nas quais poderia representar um risco a saúde. Como no hospital há varias enfermarias, foram aleatoriamente escolhidas 4 para as coletas. As cozinhas foram selecionadas por ocorrer a manipulação e armazenamento de alimentos que podem ser atrativos em potencial para as formigas. Neste ponto foram determinados 3 locais de inserção de coleta.

As formigas foram coletadas no período vespertino, em distintos pontos do hospital: Pediatria, Enfermaria e Cozinha. Coletou-se formigas utilizando iscas com mel e sardinha sobre papéis identificados, colocados em locais estratégicos nos pontos determinados. Após três horas, foram retirados e aqueles papéis que apresentavam formigas 
foram acondicionados em sacos plásticos contendo álcool 75\%. O material foi levado para o laboratório de zoologia da Faculdade de Ciências e Tecnologias de Campos Gerais e posteriormente as formigas foram separadas das iscas e mantidas em álcool 75\%, em frascos de polietileno, tipo coletor de material parasitológico, devidamente identificados.

Os materiais foram identificados em subfamílias e gênero com auxílio da chave de identificação de Baccaro (2006), no laboratório de zoologia Faculdade de Ciências e Tecnologias de Campos Gerais.

\section{RESULTADOS E DISCUSSÃO}

Nas amostragens realizadas em ambiente hospitalar no município de Campos Gerais, Minas Gerais, foram capturadas 7073 formigas, identificadas como pertencentes aos gêneros Brachymyrmex e Pheidole, que compõem as subfamílias Formicinae e Myrmicinae, respectivamente (Figuras 1, 3, 5 e 7). Na primeira coleta foram capturadas 1628 formigas pertencentes aos gêneros Pheidole e Brachymirmex (Figura 1). O maior número de espécimes (884) foi observado na cozinha, sendo $69,7 \%$ do gênero Pheidole e $30,3 \%$ Brachymirmex. $\mathrm{Na}$ enfermaria foram registrados apenas fomigas do gênero Pheidole e na pediatria não se verificou nenhum exemplar desses insetos (Figura 1).
FIGURA 1- Distribuição das formigas amostradas na primeira coleta, nos três pontos, em ambiente hospitalar no município de Campos Gerais, MG.

\begin{tabular}{lllll}
\hline Local & Tipo de isca & Subfamilia & Gênero & Número de individuos \\
\hline \multirow{2}{*}{ Cozinha 1 } & Mel & - & - & 0 \\
& Sardinha & Myrmicinae & Pheidole & 412 \\
Cozinha 2 & Mel & Myrmicinae & Pheidole & 6 \\
& Sardinha & Myrmicinae & Pheidole & 198 \\
Cozinha 3 & Mel & Formicinae & Brachymyrmex & 4 \\
& Sardinha & Formicinae & Brachymyrmex & 264 \\
Enfermaria 1 & Mel & - & - & 0 \\
& Sardinha & - & - & 0 \\
Enfermaria 2 & Mel & - & - & 0 \\
& Sardinha & Myrmicinae & Pheidole & 342 \\
Enfermaria 3 & Mel & - & - & 0 \\
& Sardinha & - & - & 0 \\
Enfermaria 4 & Mel & Myrmicinae & Pheidole & 214 \\
& Sardinha & Myrmicinae & Pheidole & 188 \\
Pediatria 1 & Mel & - & - & 0 \\
& Sardinha & - & - & 0 \\
Pediatria 2 & Mel & - & - & 0 \\
& Sardinha & - & - & 0 \\
Pediatria 3 & Mel & - & - & 0 \\
& Sardinha & - & - & 0 \\
Pediatria 4 & Mel & - & - & 0 \\
\hline Total & Sardinha & - & - & 028 \\
\hline
\end{tabular}

Fonte. Silvestre (2012).

Considerando todos os pontos de amostragem, verificou-se predominância das formigas do gênero Pheidole nesta coleta, que representaram $84 \%$ dos espécimes coletados. (Figura 2) Considerando todos os pontos de amostragem, verificou-se predominância das formigas do gênero Pheidole nesta coleta, que representaram $84 \%$ dos espécimes coletados.

Figura 2- Gêneros de formigas amostrados na primeira coleta, nos três pontos de amostragem, em ambiente hospitalar no município de Campos Gerais, MG.

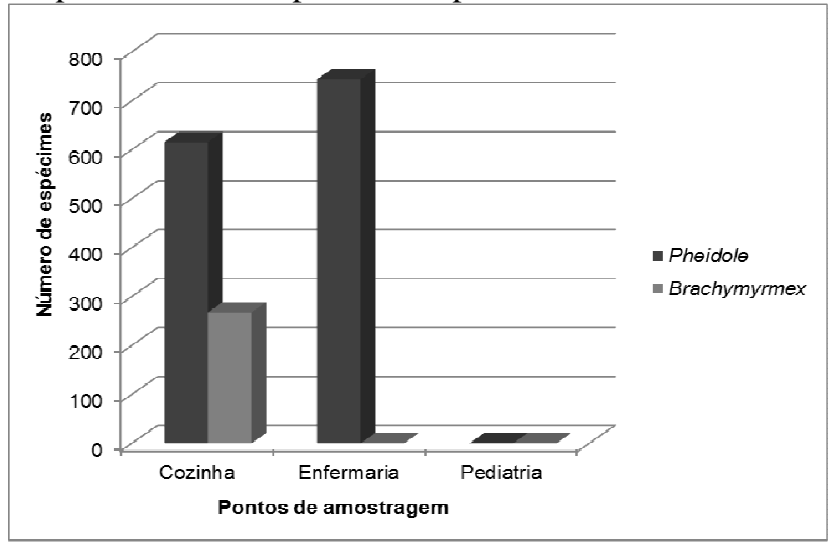

Fonte. Silvestre (2012). 
$\mathrm{Na}$ segunda coleta foram capturadas 1925 formigas (Figura 3). O maior número desses insetos foi coletado na cozinha, sendo 1422 do gênero Pheidole e 88 do gênero Brachymirmex, na enfermaria foram coletados apenas 415 espécimes do gênero Pheidole e na pediatria não se obteve amostras (Figura 4).

Figura 3 - Distribuição das formigas amostradas na segunda coleta, nos três pontos, em ambiente hospitalar no município de Campos Gerais, MG.

\begin{tabular}{|c|c|c|c|c|}
\hline Local & Tipo de isca & Subfamilia & Gênero & Nümero de indivíduos \\
\hline \multirow[t]{2}{*}{ Cozinha 1} & Mel & Mymicmae & Pheidole & 9 \\
\hline & Sardinha & Myrmicinae & Pheidole & 1196 \\
\hline \multirow[t]{2}{*}{ Cozimha 2} & Mel & Mymicimae & Pheidole & 20 \\
\hline & Sardimha & Mymicinae & Pheidole & 197 \\
\hline \multirow[t]{2}{*}{ Cozinha 3} & Mel & Formicinae & Brachymymex & 79 \\
\hline & Sardinha & Formicinae & Brachymymex & 9 \\
\hline \multirow[t]{2}{*}{ Enfermaria 1} & Mel & - & - & 0 \\
\hline & Sardinha & - & - & 0 \\
\hline \multirow[t]{2}{*}{ Enfermaria 2} & Mel & - & . & 0 \\
\hline & Sardinha & - & - & 0 \\
\hline \multirow[t]{2}{*}{ Enfermaria 3} & Mel & - & - & 0 \\
\hline & Sardimha & Mymicinae & Pheidole & 235 \\
\hline \multirow[t]{2}{*}{ Enfermaria 4} & Mel & Mymicinae & Pheidole & 3 \\
\hline & Sardinha & Mymicinae & Pheidole & 177 \\
\hline \multirow[t]{2}{*}{ Pediatria 1} & Mel & - & - & 0 \\
\hline & Sardimha & - & - & 0 \\
\hline \multirow{2}{*}{ Pediatria 2} & Mel & . & - & 0 \\
\hline & Sardinha & . & . & 0 \\
\hline \multirow{2}{*}{ Pediatria 3} & Mel & . & . & 0 \\
\hline & Sardinha & . & . & 0 \\
\hline \multirow{2}{*}{ Pediatria 4} & Mel & . & . & 0 \\
\hline & Sardinha & . & . & 0 \\
\hline Total & & & & 1925 \\
\hline
\end{tabular}

Fonte. Silvestre (2012).

Observou-se, nesta coleta, a predominância das formigas do gênero Pheidole, que representaram $95 \%$ dos espécimes coletados. A isca mais atrativa foi à sardinha que proporcionou a coleta de $94 \%$ das formigas. Ambas as iscas proporcionaram a coleta exemplares dos gêneros Pheidole e Brachymirmex.
Figura 4 - Gêneros de formigas amostrados na segunda coleta, nos três pontos de amostragem, em ambiente hospitalar no município de Campos Gerais, MG.

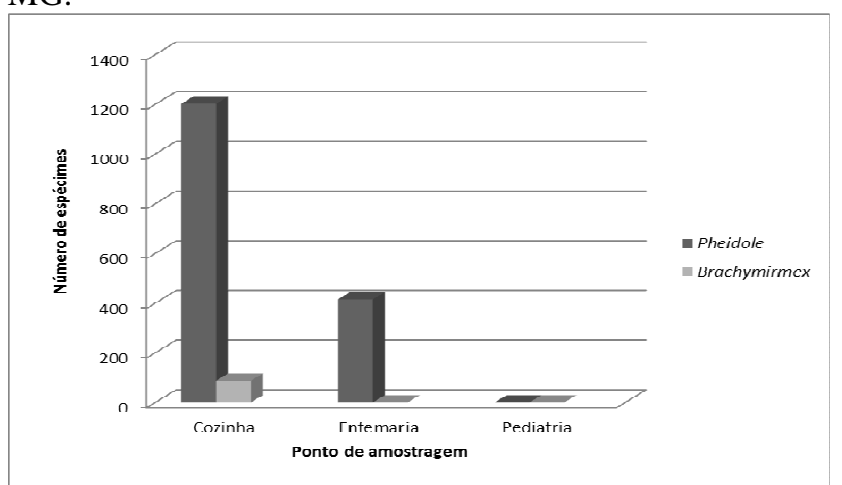

Fonte. Silvestre (2012).

Na terceira coleta, 1544 exemplares de formigas foram coletados, sendo $98 \%$ do gênero Pheidole e $2 \%$ do gênero Brachymirmex (Figura 5). O maior número de formigas foi observado na cozinha, com 1202 espécimes do gênero Pheidole e 24 do gênero Brachymirmex, na enfermaria foram observados 318 espécimes do gênero Pheidole e na pediatria nenhum inseto foi coletado (Figura 6).

Figura 5 - Distribuição das formigas amostradas na terceira coleta, nos três pontos, em ambiente hospitalar no município de Campos Gerais, MG.

\begin{tabular}{|c|c|c|c|c|}
\hline Local & $\begin{array}{l}\text { Tipo de } \\
\text { isca }\end{array}$ & Subfamilia & Gênero & Número de individuos \\
\hline \multirow[t]{2}{*}{ Cozinha I } & Mel & Myrmicinae & Pheidole & 12 \\
\hline & Sardinha & Myrmicinae & Pheidole & 798 \\
\hline \multirow[t]{2}{*}{ Cozinha 2} & Mel & Mymmicinae & Pheidole & 80 \\
\hline & Sardinha & Mymicinae & Pheidole & 312 \\
\hline \multirow[t]{2}{*}{ Cozinha 3} & Mel & Formicinae & Brachymymex & 24 \\
\hline & Sardinha & - & - & 0 \\
\hline \multirow[t]{2}{*}{ Enfermaria 1} & Mel & - & - & 0 \\
\hline & Sardinha & - & - & 0 \\
\hline \multirow[t]{2}{*}{ Enfermaria 2} & Mel & - & - & 0 \\
\hline & Sardimha & - & . & 0 \\
\hline \multirow[t]{2}{*}{ Enfermaria 3} & Mel & - & - & 0 \\
\hline & Sardinha & Mymicinae & Pheidole & 150 \\
\hline \multirow{2}{*}{ Enfermaria 4} & Mel & - & . & 0 \\
\hline & Sardinha & Myrmicinae & Pheidole & 168 \\
\hline \multirow[t]{2}{*}{ Pediatria 1} & Mel & - & - & 0 \\
\hline & Sardimha & - & - & 0 \\
\hline \multirow[t]{2}{*}{ Pediatria 2} & Mel & - & - & 0 \\
\hline & Sardinha & - & - & 0 \\
\hline \multirow[t]{2}{*}{ Pediatria 3} & Mel & - & - & 0 \\
\hline & Sardinha & . & - & 0 \\
\hline \multirow{2}{*}{ Pediatria 4} & Mel & - & - & 0 \\
\hline & Sardinha & - & - & 0 \\
\hline Total & & & & 1544 \\
\hline
\end{tabular}

Fonte. Silvestre (2012). 
Foram coletadas na terceira coleta $98 \%$ de formigas do gênero Pheidole e 2\% Brachymirmex. A isca mais atrativa foi à sardinha que proporcionou a coleta de $92 \%$ dos espécimes. Nesta coleta não foram amostrados exemplares do gênero Brachymirmex nas iscas contendo sardinha.

Figura 6 - Representação dos gêneros de formigas da terceira coleta amostradas no hospital no município de Campos Gerais MG.

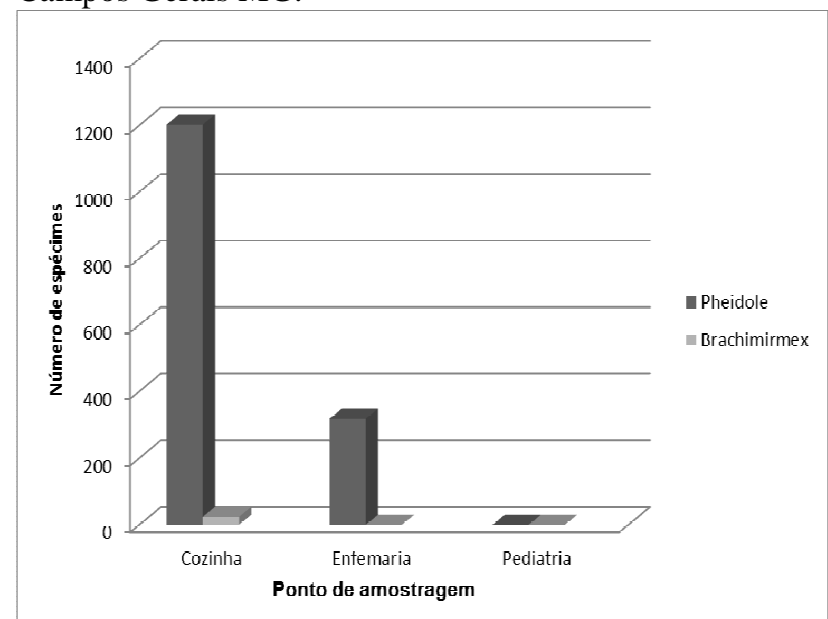

Fonte. Silvestre (2012).

$\mathrm{Na}$ quarta coleta foram capturadas 1976 formigas pertencentes aos gêneros Pheidole e Brachymirmex (Figura 7). O maior número de espécimes (1569) foi observado na cozinha, sendo 95,1\% do gênero Pheidole e 4,9\% Brachymirmex. Na enfermaria foram registrados apenas fomigas do gênero Pheidole (407 exemplares) e na pediatria não se verificou a presença de nenhuma formiga (Figura 8).
Figura 7 - Distribuição das formigas amostradas na quarta coleta, nos três pontos, em ambiente hospitalar no município de Campos Gerais, MG.

\begin{tabular}{|c|c|c|c|c|}
\hline Local & Tipo de isca & Subfamilia & Gênero & $\begin{array}{l}\text { Número de } \\
\text { indivíduos }\end{array}$ \\
\hline \multirow[t]{2}{*}{ Cozinha 1} & Mel & Myrmicinae & Pheidole & 42 \\
\hline & Sardimha & Myrmicinae & Pheidole & 1298 \\
\hline \multirow[t]{2}{*}{ Cozinha 2} & Mel & Myrmicinae & Pheidole & 42 \\
\hline & Sardinha & Myrmicinae & Pheidole & 110 \\
\hline \multirow[t]{2}{*}{ Cozinha 3} & Mel & Formicinae & Brachymyrmex & 24 \\
\hline & Sardinha & Formicinae & Brachymyrmex & 53 \\
\hline \multirow[t]{2}{*}{ Enfermaria 1} & Mel & - & - & 0 \\
\hline & Sardinha & - & - & 0 \\
\hline \multirow[t]{2}{*}{ Enfermaria 2} & Mel & - & - & 0 \\
\hline & Sardinha & - & - & 0 \\
\hline \multirow[t]{2}{*}{ Enfermaria 3} & Mel & Myrmicinae & Pheidole & 8 \\
\hline & Sardinha & Myrmicinae & Pheidole & 201 \\
\hline \multirow[t]{2}{*}{ Enfermaria 4} & Mel & - & - & 0 \\
\hline & Sardinha & Myrmicinae & Pheidole & 198 \\
\hline \multirow[t]{2}{*}{ Pediatria 1} & Mel & - & - & 0 \\
\hline & Sardinha & - & - & 0 \\
\hline \multirow[t]{2}{*}{ Pediatria 2} & Mel & - & - & 0 \\
\hline & Sardimha & - & - & 0 \\
\hline \multirow[t]{2}{*}{ Pediatria 3} & $\mathrm{Mel}$ & - & - & 0 \\
\hline & Sardinha & - & - & 0 \\
\hline \multirow[t]{2}{*}{ Pediatria 4} & Mel & - & - & 0 \\
\hline & Sardimha & - & - & 0 \\
\hline Total & & & & 1976 \\
\hline
\end{tabular}

Fonte. Silvestre (2012).

Observou durante as coleta, a predominância das formigas do gênero Pheidole, que representaram $96 \%$ dos espécimes coletados. A isca mais atrativa foi à sardinha que proporcionou a coleta de $94 \%$ das formigas. Ambas as iscas proporcionaram as coletas exemplares dos gêneros Pheidole e Brachymirmex, com exceção da pediatria que não obteve amostra nas coletas o que pode estar relacionada com o baixo numero de crianças presente sendo que duas das coletas não havia crianças presente no ambiente. 
Figura 8 - Gêneros de formigas amostrados na quarta coleta, nos três pontos de amostragem, em ambiente hospitalar no município de Campos Gerais, MG.

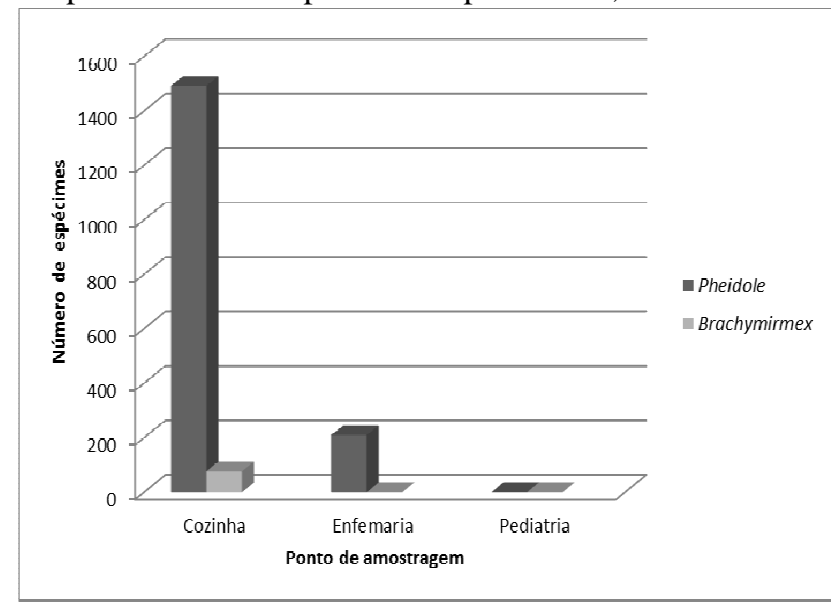

Fonte. Silvestre (2012).

Considerando todas as coletas o maior número de formigas foi encontrado na cozinha (Figura 9). Segundo Silva Jr. (1999), a cozinha é o ambiente ideal para desenvolvimento de microrganismos já que apresenta os elementos ideais para seu desenvolvimento como água, ph neutro ou acido, nutrientes, oxigênio e temperatura ao redor de $35^{\circ} \mathrm{C}$. Essas condições também são favoráveis para o crescimento, dispersão e manutenção de formigas e outros insetos denominados pragas urbanas (SCHULLER, 1999) estabelecendo uma relação intima entre os dois grupos de organismos.

Figura 9 - Distribuição das formigas amostradas nas quatro coletas, nos três pontos, em ambiente hospitalar no município de Campos Gerais, MG.

\begin{tabular}{lccc}
\hline \multicolumn{1}{c}{ Local } & Subfamilia & Gênero & Número de individuos \\
\hline Cozinha & Mymicinae & Pheidole & 4732 \\
& Formicinae & Brachymymex & 457 \\
Enfermaria & Mymicinae & Pheidole & 1884 \\
& - & - & 0 \\
Pediatria & - & - & 0 \\
& - & Pheidole & 0 \\
\hline Subtotal 1 & Mymiciciae & Brachymymex & 6616 \\
\hline Subtotal 2 & Formicinae & & 457 \\
\hline Total & & & 7073 \\
\hline
\end{tabular}

Fonte. Silvestre (2012).
O gênero mais frequentemente amostrado foi Pheidole representando 93,5\% das formigas coletadas (Figura 10). A ocorrência frequente de espécies de Pheidole em hospitais brasileiros tem sido amplamente documentada (FOWLER et al., 1993; BARROS et al. 2006; COSTA et al. 2006; FONSECA et al. 2010).

Figura 10 - Gêneros de formigas amostradas nas quatro coletas, nos três pontos, em ambiente hospitalar no município de Campos Gerais, MG.

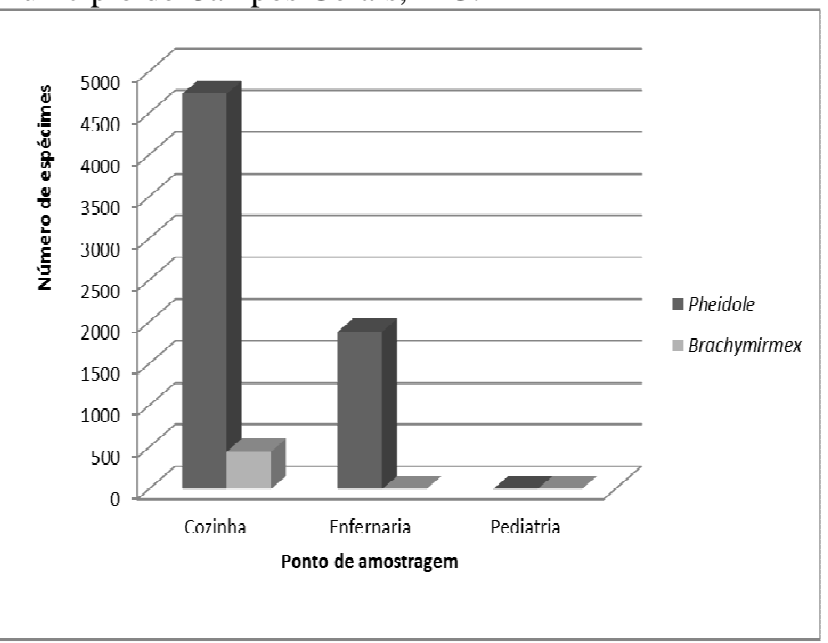

Fonte. Silvestre (2012).

O gênero Pheidole (Myrmicinae) está amplamente distribuído em diversos ecossistemas, o que lhe permite dominar os recursos alimentares e excluir de maneira eficiente seus competidores (HÖLLDOBLER; WILSON, 1990). O gênero Brachymyrmex obteve menor número de indivíduos, representando $6 \%$ dos insetos coletados. Esses resultados diferem dos obtidos por Fonseca et al. (2010) em um hospital no município de Luz, Estado de Minas Gerais, no qual o gênero mais numeroso foi Brachymyrmex, sendo que as espécies desse gênero foram encontradas tanto na área interna, 
quanto na externa. Bicho et al. (2007) também observaram formigas desse gênero em um hospital de Bagé, RS, e Silva e Loeck (1999) encontraram espécies desse gênero no interior de residências em Pelotas, RS, com elevada frequência, considerando-as inclusive como praga. No entanto, Oliveira e Campos-Farinha (2005), citam as formigas desse gênero como raras no interior de residências, sendo observadas em grandes quantidades nas áreas externas, em jardins, varandas, paredes externas das casas.

Soares et al. (2006), realizando um levantamento de formigas urbanas na cidade de Pelotas, RS, verificaram maior incidência de exemplares de Brachymyrmex sp. em locais em bom estado de conservação na cidade. Esse formicídeo tem por hábito nidificar no interior das residências utilizando cavidades em azulejos, janelas e batentes de portas e, assim, a estrutura física bem conservada das residências não impede a construção dos seus ninhos (BUENO; CAMPOS-FARINHA, 1998).

Pesquero et al. (2008), ao realizarem um levantamento de formigas em ambiente hospitalar e seu potencial como transmissoras de bactérias, ao verificaram exemplares dos gêneros Brachymyrmex sp.1 e Pheidole sp.1. Nos espécimes do gênero Pheidole em 78,4\% continham alguma espécie de bactéria patogênica e as formigas contaminadas foram observadas em todas as repartições do hospital exceto nos consultórios, berçário e sala de sutura.
A preferência dos espécimes do gênero com Brachymirmex foi pelas iscas de sardinha (Figura 11) diferente do observado em um levantamento feito por Pesquero et al.(2008) verificam que o tipo de isca oferecido proteína e carboidrato não influenciaram na atividade de forrageio desta espécie ao longo do ano apesar da aparente tendência em consumir mais açúcar durante o período de setembrojaneiro e mais proteínas entre março-agosto.

Figura 11 - Número de formigas do gênero Brachymirmex amostradas nas quatro coletas, nos três pontos, em ambiente hospitalar no município de Campos Gerais, MG.

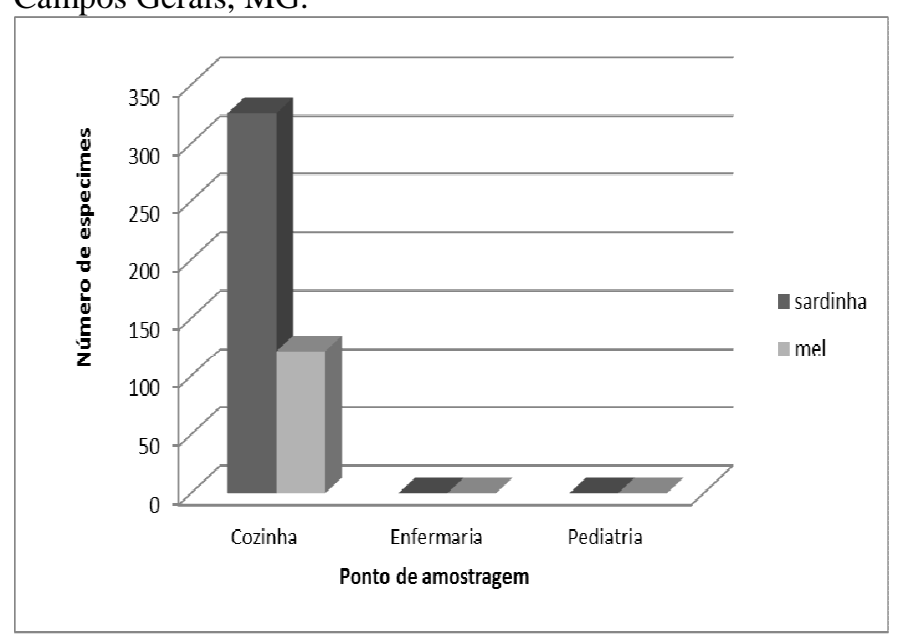

Fonte. Silvestre (2012).

As formigas do gênero Pheidole também apresentam preferência por iscas composta por sardinha (proteína) (Figura 12). Esses dados diferem dos observados no estudo de FOWLER et al. (2007) no qual as formigas Pheidole observadas não apresentaram preferência por isca de carboidrato ou proteína. 
Gráfico 7 - Número de formigas do gênero Pheidole amostradas nas quatro coletas, nos três pontos, em ambiente hospitalar no município de Campos Gerais, MG.

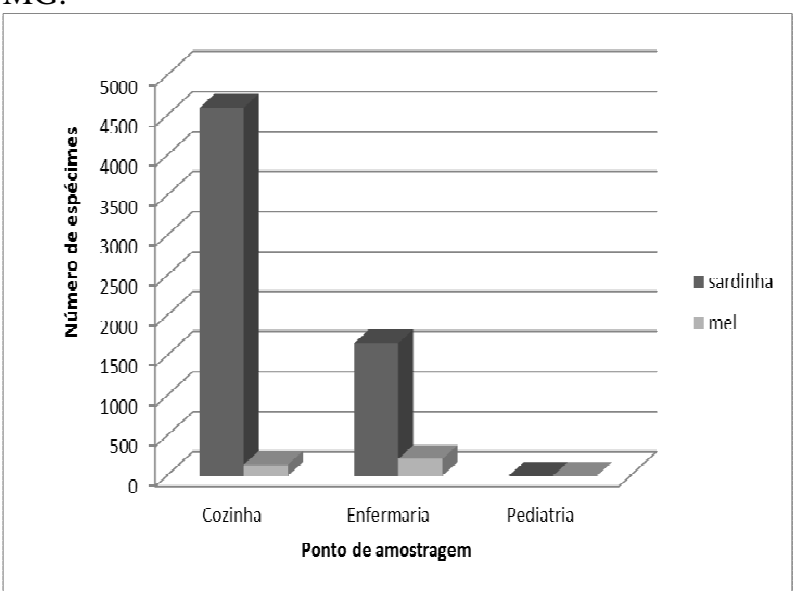

Fonte. Silvestre (2012).

Com relação às iscas utilizadas, mel (carboidrato) e sardinha (proteína), verificouse que a preferência foi por aquelas preparadas com sardinha, nas quais coletou-se $92 \%$ das formigas, em relação àquelas com mel, que propiciaram a coleta de $8 \%$ das formigas.

Estudos indicam que as formigas podem atuar como vetores mecânicos de bactérias em ambiente intra-hospitalar (BEADSON, 1972; EDWARd; BACKER, 1981; PEÇANHA, 2000). Dessa forma, como foram amostradas formigas pertencentes a gêneros já relatados como relacionados ao transporte de bactérias patogênicas, sugere-se que estudos mais aprofundados sejam realizados e também sejam estabelecidas estratégias para o controle desses insetos no hospital de Campos Gerais, MG.

\section{CONCLUSÃO}

Formigas dos gêneros Brachymirmex e Pheidole foram observadas no ambiente hospitalar no município de Campos Gerais, MG. O ponto de coleta com maior número desses insetos foi à cozinha, seguida da enfermaria e na pediatria nenhum espécime foi coletado. A isca que apresentou maior atratividade foi à composta por sardinha. Devido à possibilidade das formigas estarem associadas a microrganismos patogênicos ao ser humano e à presença de exemplares pertencentes a gêneros já relatados como vetores, ressalta-se a necessidade de estudos complementares e de estabelecer métodos adequados para o controle das mesmas nesta instituição.

\section{REFERÊNCIAS BIBLIOGRÁFICAS}

AGOSTI, D., JOHNSON, N.F. Editors. 2005.

Antbase. World Wide Web electronic

publication. antbase.org, version (05/2005).

\section{BACCARO, F.B. Chave para as principais subfamílias e gêneros de formigas (Hymenoptera: Formicidae). Instituto Nacional de pesquisa em Biodiversidade - PPBIO, Faculdades Cathedral, 2006.}

BARROS, R.A.M.; CAMPOS-FARINHA, A.E.C.; PREZOTO, F. Ocorrência, comportamento e vetoração de fungos por formigas no Hospital da Universidade Federal de Juiz de Fora, Minas Gerais. Revista Brasileira de Zoociências. n.8, p.217, 2006.

BEADSON, S. H. Pharaoh's ants as pathogens vectors in hospitals. The lancet., v. 19, n. 1. p. 425-7, 1972.

BICHO et al. Mirmecofauna (Hymenoptera, Formicidae) em hospitais e postos de saúde no município de Bagé, RS. Arquivos do 
Instituto Biológico, v.74, n.4, p.373-377, out./dez, 2007.

BUENO O. C.; CAMPOS- FARINHA A. E. C. Formigas urbanas comportamento das espécies que invadem as cidades brasileiras. Vetores \& Pragas, São Paulo, ano I, n. 2, p. 13-16, 1998.

BUENO, O. C.; CAMPOS-FARINHA, A.E. C. Formigas urbanas: estratégias de controle. Vetores \& Pragas, v.5, p.5-7, 1999.

CHEN, J. S. C.; NONACS, P. Nestmate recognition and intraspecific agression based on environmental cues in Argentine ants (Hymenoptera: Formicidae). Annals of the Entomological Society of America, v. 6, n.93, p. $1333-1337,2000$.

CINTRA, P. Ocorrência de artrópodes em ambientes hospitalares. Rio Claro:

Universidade Estadual Paulista, 2003.

CINTRA-SOCOLOWSKI, P. Histórico sobre as pesquisas com formigas em ambientes hospitalares no Brasil. Biológico, São Paulo, v.69 (supl.2), p.35, 2007.

COSTA et al. Formigas como vetores mecânicos de microorganismos no Hospital Escola da Universidade Federal do Triângulo Mineiro. Revista da Sociedade Brasileira de Medicina Tropical (online), Uberaba, v.39, n.6. 2006.

EDWARD, J. P.; BACKER, L. F. Distribuition and importance of The Pharaoh's Monomorium pharaonis (L) in national Health Service Hospitals in England. Journal of Hospital Infection v.2,p 245-254, 1981.

FERNANDEZ, F.Breve Introduccion a la Biologia Social de las Hormigas. In: FERNANDEZ, F (ed), Introducción a las Hormigas de la Región Neotropical, Capítulo 5, Bogotá, Colombia, Instituto de investigación de Recursos Biológicos Alexander Von Humboldt. 2003.
FONSECA et al. Formigas (Hymenoptera: Formicidae) urbanas em um hospital no município de Luz, Estado de Minas Gerais. Acta Scientiarum: Health Sciences. Maringá, v. 32, n. 1, p. 29-34, 2010

FOWLER, H. G.; et al. Ants as potential vectors of pathogens in hospitals in the state of São Paulo, Brazil. Insect. Sci. Applic., v.14, n.3, p.367-370, 1993.

FOWLER et al. Interação competitiva entre formigas (Hymenoptera: Formicidae) em iscas de carboidrato e proteína. Anais do VIII Congresso de Ecologia do Brasil Caxambu MG, 23 a 28 de Setembro de 2007.

GONÇALVES et al. Associação entre Formigas (Hymenoptera: Formicidae) e Bactérias Patogênicas em Cinco Hospitais do Município de Pelotas, RS. Arquivos Instituto Biológico, São Paulo, v.78, n.2, p.287-295, abr./jun., 2011.

HOLDOBLER, B., WILSON, E. O. The ants. Cambridge: Belknap Press of Harvard University Press. 1990.

LEVEY, J.; BYRNE, E M. M.. Complex AntPlant interactions: Rain Forest Ant as Secondary Dispersers and Post-Dispersal Seed Predators. Ecology, v.17, n. 6, p 1802-1812, 1993.

OLIVEIRA, M. F., CAMPOS-FARINHA, A.

E. "Formigas Urbanas do município de Maringá, PR e suas implicações". Arquivos do Instituto Biológico de São Paulo, 72 (1):33-39pp. 2005.

PASSERA, L. Characteristics of tramp species. In: WILLIAMS, D. F. (Ed). Exotic ants: biology, impact and control of introduced species. Boulder, p. 191-198, 1994.

PEÇANHA, M.P. Formigas como vetor de propagação bacteriana no conjunto hospitalar de Sorocaba- SP. 2000. Tese (Doutorado em Microbiologia Aplicada) - UNESP, Rio Claro, 2000. 
PEREIRA, R. S; UENO M. Formigas como veiculadoras de microrganismos em ambiente hospitalar. Revista da Sociedade Brasileira de Medicina Tropical, v. 41, n. 5, p. 492-495, 2008.

PESQUERO, M. A.; et al. Formigas em ambiente hospitalar e seu potencial como transmissoras de bactérias. Neotropical Entomology (online), v.37, n.4, 2008.

SHOREY, H. H. Reconhecimento da prole por operárias companheiras e não companheiras de ninho. Annais Review of Entomology, v. 18, p. 349-380, 1973

SILVA, E.J.E. ; LOECK, A.E. Ocorrência de formigas domiciliares (Hymenoptera: Formicidae) em Pelotas, R.S. Revista Brasileira de Agrociência, v.5, n.3, p.220224,1999.

SOARES, S. S.; Almeida, L. O.; Gonçalves, C. A.; Marcolino, M. T.; Bonetti, A. M. Levantamento da diversidade de formigas (Hymenoptera: Formicidae) na região urbana de Uberlândia, MG. Neotropical Entomology, 35 (3): 324-328. 2006.

SCHULLER.L. Controle de pregas nos serviços de alimentação. In: SILVA Jr., E. A. Manual de controle higiênico sanitária em alimentos. 3 ed. São Paulo livraria Varela, p. 93-102, 1999.

TANAKA, I. T; VIGGIANI, A. M. F. S; PERSON, O. C. Bactérias veiculadas por formigas em ambiente hospitalar. Arquivos médicos do ABC, São Paulo, v.32, n.2, 2007.

TEIXEIRA, M. M Formigas como carreadoras de microrganismos no hospital Escola da Universidade Federal do Triângulo Mineiro Uberaba/ MG. 2007

VITAL, M. R. Diversidade de formigas (Hymenoptera, Formicidae) em praças urbanas de Juiz de Fora, MG, Instituto de Ciências Biológicas, Juiz de Fora - Brasil Maio de 2007.
WILSON,E. O. 1971. The Insect Societies. Cambridge, Belknap. 548p.

ZARZUELA, M. F. M.; RIBEIRO, M. C. C.; CAMPOS-FARINHA, A. E. C. Distribuição de formigas urbanas em um hospital da região sudeste do Brasil. Arquivos do Instituto Biológico, v. 69, n. 1, p. 85-87, 20 